Ebisu Ebisu

Études japonaises Études japonaises

47 | printemps-été 2012

Catastrophes du 11 mars 2011, désastre de

Fukushima : fractures et émergences

\title{
Du refus à la solidarité : devant les terres dévastées
}

\section{Satoshi Kamata}

Traducteur : Jennifer Hasae

\section{(2) OpenEdition}

Journals

Édition électronique

URL : http://journals.openedition.org/ebisu/534

DOI : 10.4000/ebisu.534

ISSN : 2189-1893

Éditeur :

Institut français de recherche sur le Japon (UMIFRE 19 MAEE-CNRS), Maison franco-japonaise

Édition imprimée

Date de publication : 1 juin 2012

Pagination : 269-291

ISSN : 1340-3656

Référence électronique

Satoshi Kamata, «Du refus à la solidarité : devant les terres dévastées », Ebisu [En ligne],

47 | printemps-été 2012, mis en ligne le 03 avril 2014, consulté le 01 mai 2019. URL : http:// journals.openedition.org/ebisu/534 ; DOI : 10.4000/ebisu.534 


\section{Du refus à la solidarité Devant les terres dévastées*}

KAMATA Satoshi 鎌田慧

\section{Quarante ans de mouvements antinucléaires}

Ma première enquête sur un mouvement d'opposition en lien avec le nucléaire, c'était à Rokkasho-mura 六ヶ所村, sur la presqu'île de Shimokita 下北. Le gouvernement venait de rendre public le projet d'y construire un gigantesque combinat pétrolier dans le cadre de son Nouveau Plan national d'aménagement du territoire (Shin zenkoku sōgō kaihatsu keikaku 新全国 総合開発計画) de 1969. Mon enquête portait sur l'état naissant du mouvement d'opposition à la pollution. Les problèmes d'asthme et de pollution marine provoqués par les combinats de Yokka-ichi 四日市 et de Kawasaki

\footnotetext{
- Kamata Satoshi est un journaliste né en 1938 à Hirosaki. Ouvrier dans une imprimerie à l'âge de 18 ans, il est licencié après avoir voulu y créer un syndicat. Il suit alors des cours de littérature russe à l'université Waseda et devint par la suite journaliste d'investigation. Il est l'auteur de très nombreux ouvrages sur les questions de société, comme la pollution, les conditions de travail, l'ijime (brimades, intimidations, harcèlements), et de biographies de révolutionnaires comme Ōsugi Sakae (1885-1923). Il a obtenu plusieurs prix, dont le Mainichi shuppan bunka-shō pour son ouvrage sur le mouvement d'opposition à l'installation de l'usine de retraitement des combustibles nucléaires, Rokkasho-mura no kioku (La mémoire de Rokkashomura). Certains ont été traduits dans plusieurs langues. Il est notamment l'auteur de Toyota, l'usine du désespoir (1973, réédition 2008), traduit en français en 1976 [N.D.L.R.].

* Ce témoignage est la traduction d'un article publié dans la revue Gendai shisō 現代 思想, 39-14 : 34-45, octobre 2011.
} 
川崎 commençaient à faire l'objet de protestations. À Rokkasho-mura, les problèmes de pollution constituaient aussi le point de départ du mouvement.

Le projet d'aménagement s'étendait sur une surface de 15000 ha ( 5000 ha aujourd'hui) et faisait peser une autre menace, celle de la disparition du village. Le mouvement était donc dirigé contre les expropriations. Il ne s'agissait pas d'un mouvement antinucléaire, car la nature exacte du projet n'était pas clairement dévoilée. En effet, comme je l'ai écrit dans Rokkasho-mura no kiroku 六ヶ所村の記録 (Chroniques de Rokkasho-mura, 1991), le nucléaire ne suscitait pas de débat à l'époque : les dirigeants économiques accordaient le primat au développement industriel. Je partais aussi, à l'époque, faire des reportages sur les luttes d'habitants. Là, je distribuais une brochure que j'avais conçue, "Pour l'arrêt du projet " (Kaihatsu soshi no tame ni 開発阻止のために), en soutien aux mouvements d'opposition aux expropriations.

Des mouvements contre la pollution et contre les expropriations sur lesquels je menais des enquêtes à partir de la fin de l'année 1969 et durant les années 1970, j'en suis arrivé à traiter directement du problème du nucléaire lorsque, en 1973, je me suis intéressé au mouvement d'opposition à la construction de la centrale de Kashiwazaki-Kariwa 柏崎・刈羽1 dans le département de Niigata. Au départ, j'étais parti sur le terrain dans la continuation de mes deux autres enquêtes sur les luttes contre la pollution ${ }^{2}$.

Un mouvement similaire à celui de Kashiwazaki-Kariwa existait à la même époque dans la ville d'Ikata 伊方, dans le Shikoku, dont les habitants

1. La centrale nucléaire de Kashiwazaki-Kariwa est située à cheval entre la ville de Kashiwazaki et le village de Kariwa. Son projet de construction a été rendu public par Tepco en 1969, son premier réacteur mis en service en 1985. Cette centrale est devenue la plus puissante au monde depuis la mise en service de son septième réacteur en 1997 [N.D.T.].

2. Ces enquêtes avaient donné lieu à deux livres : l’un, Kakusareta kōgai 隠された公害 (Cette pollution cachée, 1970) sur la maladie itai itai byōイ夕イイ夕イ病 à Tsushima 対馬; l'autre, Shi ni taeta fükei 死に絶えた風景 (Un paysage disparu à jamais, 1971) sur la baie de Dōkai 洞海 dans le nord de Kyūshū qui, avec l'installation de l'usine métallurgique de Yawata seitetsu 八幡製鉄 depuis la fin de l'époque Meiji, avait progressivement été remblayée par la construction de terre-pleins et polluée. Dans ces livres, je traitais du développement industriel, de la pollution et des mouvements d'habitants. 
constituaient le fer de lance. Ces deux grandes luttes antinucléaires se résumaient dans l'expression qui courait à l'époque : "Kariwa à l'est, Ikata à l'ouest "(higashi no Kariwa, nishi no Ikata 東の刈羽、西の伊方). D'autres mouvements surgirent ailleurs, mais de moindre importance et furent muselés l'un après l'autre et ne purent atteindre une quelconque ampleur. C'est pour répondre à la nécessité de relayer les informations sur ces mouvements que je suis allé à Kariwa, puis à Ikata, et que j’ai poursuivi mon enquête sur d'autres mouvements antinucléaires.

Pourquoi les habitants s'opposaient-ils à l'installation de centrales nucléaires ? Bien sûr, c'était la conscience, dès cette époque, des dangers du nucléaire. Vers 1971, on commençait à trouver au Japon des textes écrits par des scientifiques américains comme John William Gofman, dont certains présentés dans la revue Gijutsu to ningen 技術と人間 (L'homme et la technique). On trouvait aussi des critiques émanant de spécialistes du nucléaire, comme l'ouvrage de Zhores A. Medvedev sur le désastre de Kyshtym², Uraru no kaku sanji ウラルの核惨事 (Le désastre nucléaire de l'Oural). Nous avions aussi, au Japon, des critiques formulées par des scientifiques comme Kume Sanshirō 久米三四郎 ou Mito Iwao 水戸巌, ou encore Anzai Ikurō 安斎育郎 qui menait une recherche sur les conséquences des radiations sur la végétation. Ces auteurs nous fournissaient un appui théorique. M. Kume parcourait tout le Japon ; quant à M. Mito, je l'avais présenté au mouvement de la ville de Mutsu むつ qui s'opposait au navire expérimental à propulsion nucléaire du même nom. J'avais également demandé au journaliste scientifique Hoshino Yoshirō 星野芳郎 de se joindre à une assemblée organisée par la coopérative de pêcheurs qui protestait contre le navire Mutsu. Je suis ainsi allé de Rokkasho-mura à Higashidōri-mura 東通村, puis à Mutsu, puis à Ōma 大間 4 surtout ces dix dernières années, et dans de nombreuses autres localités encore.

3. Le désastre de Kychtym est un accident nucléaire de niveau 6 qui s'est produit le 29 septembre 1957 au complexe nucléaire Maïak, une usine de retraitement de combustible nucléaire située près de la ville de Kychtym en Union soviétique, dans l'actuelle Russie [N.D.T.].

4. Toutes ces localités sont situées sur la presqu'île de Shimokita, dans le département d'Aomori [N.D.T.]. 
Dans les années 1970, les protestations contre les expropriations et contre la renonciation aux droits de pêche étaient au cœur des luttes d'habitants. Dans les années 1980, est venu s'ajouter le problème des enquêtes publiques manipulées, toujours d'actualité. Une fois rachetés les terrains et les droits de pêche, puis la centrale nucléaire construite, on organisait avant sa mise en service un débat public pour informer les habitants, mais il était manipulé5. Il y a eu quelques attaques en justice contre ces pratiques mais elles n'ont jamais eu gain de cause. Les centrales ont été ainsi mises en activité.

Malgré ces conditions, les mouvements d'opposition se sont maintenus. Ils ont repris un nouvel élan lors de l'accident de Three Mile Island, puis surtout avec Tchernobyl. Mais avec le temps, ces élans se sont taris.

En fait, nous avons tous - y compris les antinucléaires, y compris moimême - fini par reconnaître l'existence du nucléaire. Bien sûr, nous n'adhérons pas à cette situation, mais nous nous en accommodons.

Mon premier livre sur le nucléaire a été écrit il y a trente ans (1982), le dernier, qui sortira bientôt ${ }^{6}$, rassemble des chroniques dont la dernière a été publiée six mois avant l'accident de Fukushima. Je n'ai cessé de relayer les mouvements et les voix protestataires, et les réunions d'opposants n'ont jamais cessé. Et pourtant, le mouvement antinucléaire manquait d'impact populaire. Désormais Higashidōi-mura était presque le seul site où rien n'était encore construit. Partout ailleurs, les résistances étaient organisées contre les projets d'agrandissement de centrales déjà en service. À Ikata, le mouvement d'opposition a réussi à faire interdire un tel projet d'agrandissement en portant l'affaire en justice. L'accident du réacteur de Monju もんじゅ ${ }^{7}$, où les travaux de réparation ont été inefficaces face aux pannes successives, et le grave accident nucléaire de la compagnie JCO à Tōkai-

5. Il s'agit de débats organisés par les organes du gouvernement avec la complicité de la compagnie d'électricité, où, par exemple, les habitants qui pouvaient poser des questions étaient choisis en amont en fonction de leurs opinions par rapport au nucléaire, ou encore les questions-réponses préparées en amont par la compagnie d'électricité qui les donnait ensuite à l'agent de l'État chargé d'animer le débat [N.D.T.].

6. Rupo : Shimokita kaku hantō ルポ——下北核半島 (Reportage sur la presqu'île nucléaire de Shimokita), 2011.

7. Réacteur à Tsuruga 敦賀 dans le département de Fukui, mis en marche en 1994, mis à l'arrêt en 1995 à la suite d'une fuite de sodium ayant provoqué un incendie. La remise 
mura 東海村 ${ }^{8}$ n'ont pas suscité de regain du mouvement antinucléaire dans la population.

Telle était la situation lorsque l'accident nucléaire de Fukushima s'est produit. Dans le passé, nombreux furent ceux qui, tout en se disant opposés à la guerre du Pacifique, n'ont rien pu faire. J'ai le sentiment amer que cette histoire se répète. Nous n'aurons aucune excuse à présenter face aux reproches des générations suivantes. Nos mouvements étaient trop faibles, nous sommes passés inaperçus auprès de la population. Nous sommes face aux mêmes problématiques que lorsqu'il s'agissait d'agir contre la guerre. Cette fois-ci, il ne faut donc pas manquer le rendez-vous.

\section{La férocité de la logique de l'argent}

Le mouvement antinucléaire n'a pas perdu la bataille sur le plan des arguments : la critique du nucléaire est juste, l'accident de Fukushima est venu malheureusement le prouver. Le mouvement a toutefois perdu, face au mensonge. Car tout le nucléaire est fondé sur le mensonge, c'est-à-dire sur une fiction. Il suffisait de démolir la logique adverse pour gagner la bataille contre le nucléaire, mais notre mobilisation a été trop faible. Si on a gagné sur le plan des arguments, on a perdu sur le plan de la mobilisation. On n'a donc rien gagné.

Le discours promu par les pro-nucléaires était celui de la " sécurité ", le nôtre celui du "danger ". Dans cet affrontement entre la "sécurité » et le " danger ", ils ont toujours accolé l'adjectif " absolu », ce qui donnait " sécurité absolue ». Or, l’argument de la « sécurité absolue " s’avère plus

en marche initialement prévue en 2008 sera effective en 2010. Quelques mois plus tard, un autre accident oblige à l'arrêt du réacteur [N.D.T.].

8. Grave accident de criticité (fissions provoquées par une réaction en chaîne incontrôlée) survenu en 1999 dans l'usine de production d'uranium enrichi de la compagnie JCO, filiale du trust Sumitomo, à Tōkai-mura, obligeant la population à être évacuée dans un rayon de $200 \mathrm{~m}$ et à se confiner dans un rayon de $10 \mathrm{~km}$. Cet accident a été classé au niveau 4 des accidents nucléaires. Deux des trois employés les plus gravement exposés aux radiations sont décédés peu après. Au total, plus de 400 personnes ont été exposées à des doses de différents niveaux. (Sources : <www.oecd-nea.org/law/nlbfr/ nlb-66/014_022.pdf et http://www.acro.eu.org/tokaimura.html>) [N.D.T.]. 
fort que celui du "danger absolu ", car une fois démontré le danger, il est difficile de trouver un argument qui vienne renforcer cette idée de danger. Tandis que dans leur cas, pour appuyer l'argument de la "sécurité absolue ", ils recourent à l'argument qu'en dernière instance, il existe un garant responsable. Je l'ai souvent répété, la réplique des responsables des zones d'implantation de centrales nucléaires a toujours été celle-ci : "L'État dit que c'est absolument sûr ". Si l'État garantit ainsi la sécurité de manière absolue, il n'y a plus qu'un État étranger capable de trancher, par la guerre, en accusant la situation de dangereuse. La question ne peut plus être réglée qu’à ce niveau-là. Dans cette bataille " sécurité " contre "danger ", les pro-nucléaires s'octroient la garantie suprême par le recours au pouvoir de l'État. Les antinucléaires n'ont pas la caution de ce dernier. Nous avons au mieux celle de quelques individus, ce qui ne fait pas le poids dans le rapport de force.

À cette caution de l'État, s'ajoute un fonds exorbitant issu de ses caisses, la "Subvention provenant des trois lois sur l'énergie électrique " (Dengen sanpō kōfukin 電源三法交付金). Une collectivité locale reçoit en effet près de 100 milliards de yens pour chaque réacteur d'une puissance de 1350 mégawatts (ce qui équivaut à la puissance des plus grands réacteurs actuels) qu' elle accepte de construire. Il y a d'abord une subvention qui est versée durant dix ans à partir de la prise en charge de la construction par la collectivité locale et qui s'élève à près de 48 milliards de yens; s'y ajoutent ensuite 50 milliards de yens qui comprennent la subvention allouée à partir de la mise en activité du réacteur et la taxe foncière. Cette dernière, cependant, diminue progressivement et se tarit au bout de vingt ans. L'argent devient une drogue, voilà comment une collectivité locale finit par construire un autre réacteur.

Tout cet argent provient des factures d'électricité, dont le calcul des tarifs comprend à la fois le coût de construction des réacteurs et celui du fonctionnement. De plus, l'État verse 100 milliards de yens pour soutenir le développement local. Que l'État aide une entreprise privée, cela ne s'est vu nulle part ailleurs que dans le secteur nucléaire. Jamais l'État ne donnerait de l'argent à Toyota pour la construction d'une usine ! Les centrales nucléaires ont ainsi été construites dans le cadre d'une politique nationale.

Le mouvement antinucléaire ne peut que perdre face à un tel pouvoir de l'argent. 
L'argent ainsi usurpé aux consommateurs par le biais des factures d'électricité vient renflouer les caisses des grandes entreprises générales de construction (zenekon ゼネコン) qui construisent les centrales en montant des joint-ventures. Les entreprises locales dans ces joint-ventures ne sont que des sous-traitants, ou des sous-traitants de sous-traitants. Les zenekon profitent encore, cette fois de l'argent public, en construisant dans ces localités divers équipements coûteux, et subventionnés par l'État ${ }^{9}$, et autres infrastructures sans rapport direct avec les centrales. Cet argent gagné par les zenekon part ensuite dans les fonds politiques levés par les hommes politiques. Autrement dit, l'argent circule en vase clos.

Les compagnies d'électricité n'ayant rien à leur charge, les dépenses sont sans limites. Le "J Village ", le complexe sportif de l'Association japonaise de football, en est un exemple emblématique : un coût de 16 milliards de yens pour sa construction, un don de la compagnie d'électricité ${ }^{10}$. À Mutsu, la rénovation du centre commercial désaffecté a aussi bénéficié d'un don de la compagnie d'électricité s'élevant à 1,6 milliards de yens pour être transformé en un immense hôtel de ville.

Même après l'accident de Fukushima, de nombreux hommes politiques, proches du ministère de l'Économie, du Commerce et de l'Industrie (le METI) et du ministère des Finances, à commencer par le Premier ministre Noda 野田, continuent d'affirmer que, du point de vue économique, l'activité des centrales est appropriée. Sans l'énergie nucléaire, menacent-ils, toute l'industrie japonaise en pâtirait, les usines seraient délocalisées, les tarifs de consommation d'électricité augmenteraient.

Il n'y a finalement que la logique de l'argent. Deux logiques antagonistes, celle de la vie et celle de l'argent, se font face, mais la vie est réduite à celle d'une minorité. La radioactivité continue de se propager dans tout le pays, mais dans l'immédiat, on met en balance la vie des riverains de Fukushima Daiichi avec la prospérité de la population sur tout le territoire national. Que les centrales continuent d'être en service n’a évidemment que peu de

9. L'auteur désigne ici les constructions d'apparat : il s'agit d'équipements ou d'infrastructures publics prestigieux (bibliothèques, musées, ouvrages de génie civile, etc.), dont les travaux coûtent chers, mais dont l'utilité est contestée et l'entretien très onéreux [N.D.T.].

10. Il s'agit de Tepco [N.D.T.]. 
répercussions sur le niveau de richesse de la majorité de la population. Mais nous sommes tellement pris par la logique utilitariste qui préfere la prospérité de la majorité aux dépens de la mort d'une minorité que même avec un accident d'une telle envergure, on s'inquiète davantage de l'économie que de la vie des hommes. Nous n'arrivons toujours pas à annihiler cette avidité pour l'argent.

Ces mêmes problèmes se retrouvent au niveau local. En gros, on arrose les opposants de bons repas pour les neutraliser ou on les amadoue avec des perspectives de débouchés pour leurs enfants et des créations d'emplois sur les chantiers. Les questions d'argent priment, même en cas d'accident. À la centrale d'Ōma 大間, comme je l'ai décrit dans La presqu'île nucléaire de Shimokita (2011), quand le chantier s'interrompit pour cause d'accident, les entreprises locales, privées de commandes, réclamèrent la reprise du chantier. La même logique est à l'œuvre chez le Premier ministre Noda. Seule compte la réussite économique, et la reprise de la construction ou de l'activité de la centrale est exigée. Les mouvements antinucléaires n’ont pas réussi jusqu’à présent à rompre cette logique.

L'argument du danger que nous utilisions jusqu'à présent ne peut être démontré qu'avec un accident. Nous sommes alors contraints à un débat très difficile. Même si le mouvement antinucléaire reprend de la vigueur avec un accident grave comme celui-ci, du temps va s'écouler avant un prochain accident. Pendant ce temps, les promoteurs du nucléaire vont définir cet accident comme un accident particulier dû à une déficience propre à la centrale de Fukushima. Face à cela, un argument basé sur un prochain accident ne peut gagner. En somme, comment, avec l'argument de la vie qui relie le passé, le présent et l'avenir - pourrions-nous faire émerger une résistance contre la dangerosité des accidents et contre la priorité donnée à l'économique ? Jusque là, nous n'arrivions pas à construire ce discours.

\section{Penser le refus}

Que devons-nous retenir de ces mouvements antinucléaires? Il faut " penser le refus ". Dans mon article publié dans la revue Sekai 世界 (novembre 2011), j'évoque l'exemple de M. Masukura Takashi 舛倉隆, l'opposant à la centrale de Namie 浪江, pour illustrer le sens de ce "refus ». Sa pensée du refus est capitale. Elle consiste à dire que, comme les gens de la compagnie 
d'électricité sont des dupeurs professionnels, je n'accepte pas de négocier avec eux. En tant que stratégie de combat, elle s'est avérée très efficace. On dit souvent qu' " en démocratie, on doit aussi écouter ses ennemis ", mais il n'y a aucun débat possible, même scientifique, avec ceux qui veulent construire les centrales. Tout ce que savent faire les promoteurs du nucléaire lorsqu'une situation est bouchée, c'est de museler les opposants avec de l'argent. Avec des "il faut écouter ses adversaires ", "faites des contrepropositions ", on ouvre la porte à cette négociation.

Certes, il existe des cas de combats menés grâce aux débats. Le mouvement contre la construction du barrage mobile de Daijū sur la rivière Yoshino (Yoshino-gawa Daijū zeki 吉野川第十堰), dans le département de Tokushima, en est un exemple. Il s'agit d'un mouvement de citoyens unique dans son genre qui a gagné contre le ministère de la Construction ${ }^{11}$ et a réussi à barrer le projet. Il y a aussi le mouvement contre la centrale nucléaire de Maki 巻 qui a gagné, non par le débat, mais par la persévérance $^{12}$. Il s'agit là d'une victoire obtenue grâce à l'inventivité des citoyens. Mais en général, il est très difficile de gagner par la parole contre la construction d'une centrale. Une compagnie d'électricité est un adversaire féroce, tenace, doté de beaucoup d'argent et offensif. Dans le cas du barrage de la rivière Yoshino, les adversaires étaient les bureaucrates du ministère de la Construction. Comme ces derniers changent de poste au bout d'un certain temps, leurs offensives n'étaient pas très virulentes. Qu'en était-il dans le cas de la centrale de Maki ? L'adversaire n'était pas la compagnie d'électricité de Tokyo, Tepco, mais celle du Tōhoku (Tōhoku denryoku 東北電力), ce qui explique peut-être sa faiblesse. L'adversaire de M. Masukura à Namie était également la Tōhoku denryoku.

Cependant, dans une rétrospective du passé, la « pensée du refus » m’apparaît précieuse. On dit : "Le refus, ce n'est pas un raisonnement, c'est émotionnel et c'est têtu, il n'y a rien à en tirer ", mais c'est justement ce

11. L'actuel ministère du Territoire, de l'Equipement, des Transports et du Tourisme [N.D.T.].

12. Il s'agit d'un projet de construction d'une centrale nucléaire par la compagnie d'électricité Tōhoku denryoku dans la ville de Maki, département de Niigata, lancé en 1971. Les opposants au projet, après plusieurs années de lutte et de tentatives de voir leur candidat élu aux municipales, ont réussi à organiser en 1996 un référendum populaire qui, avec $61 \%$ des voix contre, a permis de rejeter le projet [N.D.T.]. 
qui fait sa force contre l'ennemi. Or, nous devons repenser nos moyens d'action. Dans cette réflexion, il y a un mouvement qui m'a influencé, celui qui s'est constitué contre la construction de la base militaire américaine à Henoko 辺野古 sur l'île d'Okinawa. Il s'agit d'un " mouvement de refus " total. Il nous montre que le refus est la seule modalité de lutte possible lorsque le projet auquel on s'oppose est à ce point avancé. Peut-être qu'à l'avenir, M. Nakaima 仲井真, le préfet d'Okinawa, sera amené à faire des compromis politiques, mais les habitants de Henoko, les grands-pères et les grands-mères, les gens qui les ont soutenus, n'en ont fait aucun durant ces quinze dernières années, depuis l'accord $\mathrm{SACO}^{13}$. Le gouvernement ne sachant plus quoi faire a essayé de recourir à toutes sortes de ruses en accumulant des indemnisations pour concilier et amadouer les habitants, sans succès. Pour s'opposer au transfert de la base dans leur ville, les habitants ont pendant toutes ces années continué à organiser tous ensemble des sit-in actifs sur la plage. Cette idée-là, je suis de plus en plus persuadé qu'il faut la réévaluer à sa juste valeur. Voilà la conclusion à laquelle je parviens aujourd'hui, après toutes ces nombreuses années d'observation des mouvements.

À la réflexion, c'est aussi armée de cette "pensée du refus » que Kumagai Asako 熊谷あさ子 protestait contre la construction de la centrale d’Ōma, que Koizumi Kingo 小泉金吾 s'opposait au projet de Rokkasho-mura. Il est important de savoir que, à la manière des " hommes illustres " dont on lit les «Vies », les « penseurs du refus » n'ont jamais cessé d'exister et de tracer

13. Il s'agit d'un accord conclu entre le Japon et les États-Unis en 1996, basé sur le rapport final du Comité d'action spéciale sur Okinawa (Special Action Committee on Okinawa, SACO) dont l'objectif était d'étudier les moyens de réduire la présence des bases américaines sur l'île d'Okinawa. Le rapport établissait 27 recommandations relatives à la restitution des bases et des équipements, le déplacement des lieux d'entraînement militaire, la réduction des nuisances sonores. Cet accord qui devait aboutir à la restitution de $21 \%$ de la superficie alors occupée par les bases prévoyait notamment le déplacement de la base aérienne de Futenma 普天間 dans les 5 à 7 ans à venir. En 1999, un plan voit le jour qui prévoit de construire une piste sur la mer de 1500 mètres en face du village de Henoko, soulevant un grand mouvement de protestations. Depuis 2004, les études de faisabilité étant en cours malgré le refus exprimé par les habitants via un référendum local, ces derniers font un sit-in continu pour empêcher la réalisation du plan [N.D.T.]. 
le fil de l'histoire. Dès que vous refusez une fois, on vous traite de têtu et d'imbécile, et vous finissez peu à peu par être isolé de la communauté locale et des voisins. On vous dit que vous êtes obstiné et que vous n'écoutez personne. Mais si on arrive à persévérer dans cette idée, qu'obtient-on au final ? Dans le cas de la centrale de Maki, tous les terrains ont fini par être expropriés, à l'exception des terrains municipaux. Un opposant au projet, lorsqu'il fut élu maire de la ville de Maki, a eu l'idée de vendre ces derniers aux habitants qui s'y opposaient également. Normalement, on ne vend pas les biens communs d'une ville à des personnes privées. Ici, la bataille a ainsi été emportée par une action inédite. Il existe donc des combats qui ont été gagnés par la persévérance d'un seul individu, malgré son isolement.

Il est vrai, dans d'autres cas, celui-ci a mené à la défaite. C'est le cas par exemple de la lutte de Sanrizuka (Sanrizuka tōsō 三里塚闘争). Pour le moment, la piste d'atterrissage de 2500 mètres prévue à l'aéroport de Narita est inachevée car il reste encore quelques riverains qui s'y opposent. Leurs maisons étant situées à l'une des deux extrémités de la piste, celle-ci a été prolongée à l'autre extrémité faute de mieux. C'est quelque part une défaite pour les opposants, mais ils continuent de résister. Pour l'un d'eux, M. S., qui habite pile en dessous de la ligne aérienne, sa vie est une vraie torture, sous un assaut continu de bruits assourdissants. Là, ce n'est plus que de l'obstination. Il pense lui-même avoir perdu, tellement sa vie est devenue infernale, mais il ne se soumet toujours pas. Comment estimer un tel état d'esprit?

En somme, dans l'histoire des mouvements populaires japonais, je pense que nous devons réserver une place particulière à ces personnes qui ont lutté jusqu'à leur mort par le refus. Écrire «Les Vies des penseurs illustres du refus » qui relierait toutes ces personnes est un énorme travail que je ne pourrai peut-être plus assurer maintenant, mais c'est l'idée que j'avais en tête en écrivant l'article publié dans la revue Sekai.

En fin de compte, les mouvements d'opposition au nucléaire ne pouvaient s'appuyer que sur la logique du refus. Il n'y avait aucune place pour le compromis. Les habitants des zones de construction de centrales nucléaires n'ont ni accepté des compromis favorables aux intérêts de l'État, ni perdu sur le plan des arguments. Ils ont perdu face à l'immense pouvoir de l'argent et face aux divers avantages qu'on leur a offerts. Les enfants des opposants se voyaient proposer les bons offices de la compagnie d'électricité pour trouver un emploi. Les zones de construction des centrales sont 
des zones dépeuplées. La faible densité démographique était en effet une condition clairement exigée pour que l'Agence pour les Ressources naturelles et l'Énergie (Shigen enerugī chō 資源エネルギー庁) du METI donne son autorisation. Dans ces zones, il n'y a pas d'emploi à vie et l'existence y est difficile. Elles capitulent devant l'argent ou d'autres avantages. Ne faut-il pas revaloriser cette "pensée du refus » avec laquelle, malgré ces conditions difficiles, des personnes poursuivent leur combat? Voilà aujourd'hui mes préoccupations.

Il existe une "Carte des centrales nucléaires " (Genpatsu chizu 原発地図) qui indique les sites où les centrales ont été construites et que j'ai reproduite dans mon livre Nihon no genpatsu kiken chitai 日本の原発危険地帯 («Les zones nucléaires dangereuses du Japon », 2011). C’est une drôle de carte : elle signale les ravages de la défaite mais les victoires n'y laissent aucune marque $^{14}$. Plus de quarante ans sont passés depuis la mise en service de la centrale de Fukushima Daiichi en 1970 ; les lieux où les mouvements d'opposition ont gagné depuis ont été effacés de la carte, n'y sont indiqués que les lieux où ils ont été battus. Pourtant, dans certaines localités qui ont perdu, des protestations individuelles ont existé, dans d'autres, elles se poursuivent encore aujourd'hui, empêchant la réalisation du projet. Comment rendre compte de ces réalités ?

À la centrale d'Ōma, on a obtenu la modification du projet pour déplacer le cœur du réacteur. À Higashidōri-mura ${ }^{15}$, le projet de construction de la centrale avait été décidé en 1965. Or il aura fallu plus de trente-cinq ans pour que la centrale voie le jour. C'est inimaginable pour tout autre type de projet d'industrialisation. Pour une centrale en revanche, ils sont prêts à prendre le temps nécessaire à l'élaboration des conditions qui finiront

14. Pour les sites qui ne figurent pas sur la carte, l' "Almanach de la sortie du nucléaire " (Datsu genpatsu nenkan 脱原発年鑑, 2011) édité par le Centre citoyen d'information sur le nucléaire (Genpatsu shiryō jōhō shitsu 原発資料情報室, abrégé CNIC en anglais) permet de se faire une idée des localités où le projet de construction d'une centrale a complètement disparu, comme dans la ville de Suzu 珠洲 dans la presqu'île de Notō 能 登 ou dans la ville de Maki dans le département de Niigata, où les habitants mènent une vie paisible.

15. Où il y a aussi un immense terrain qui a été racheté et dont on ne sait pas encore s'il sera destiné à une usine de retraitement [N.D.T.]. 
par satisfaire les opposants et les soumettre au compromis. Au départ, les opposants protestent contre la pollution qui sera générée par l'installation d'une grande industrie. Mais le temps passant, la création d'emplois ou la revitalisation de l'économie locale sont des arguments qui viennent voiler les problèmes de pollution. Aujourd'hui, bien que les projets de nouvelles installations concernent moins les industries lourdes que les industries de l'informatique, celles-ci contribuent malgré tout à la création locale d'emplois. A contrario, les centrales n'ont aucune répercussion sur l'emploi local. À chaque contrôle périodique, une centrale rassemble près de 3000 travailleurs, mais ces derniers viennent de tout le Japon et repartent aux quatre coins du pays aussitôt le contrôle terminé. Une centrale n'apporte donc ni emploi, ni développement local. C'est pourquoi seule la "pensée du refus » est viable.

\section{Créer un circuit solidaire}

Seulement, le refus signifie l'isolement. Se pose donc la question du rapport entre isolement et solidarité. Dans un premier temps, nous devons faire connaître l'existence parmi la population de ces personnes isolées qui se sont battues. Cela amènera ensuite à réfléchir sur la manière dont nous pouvons construire une solidarité.

Les mouvements portés par les différents groupes de personnes victimes des radiations peuvent être de bons exemples sur lesquels appuyer nos réflexions. M. Ōishi Matashichi 大石又七, qui était membre de l'équipage du navire Daigo Fukuryū-maru 第五福龍丸 ${ }^{16}$, a rejoint le mouvement antinucléaire. Ne pourrait-on pas faire émerger de nouvelles questions en tissant des liens non seulement entre les victimes de la contamination de Fukushima, de Hiroshima, de Nagasaki, du Daigo Fukuryū-maru, mais aussi les victimes des essais nucléaires du Pacifique Sud, les travailleurs contaminés des mines d'uranium partout dans le monde, les victimes de Tchernobyl ? Cela signifie que, puisque le mouvement pour l'abolition de l'arme atomique

16. Thonier japonais dont l'équipage a été contaminé par les retombées radioactives de l'essai d'une puissante bombe $\mathrm{H}$ par les États-Unis, dans l'atoll de Bikini, le $1^{\text {er }}$ mars 1954 [N.D.T.]. 
(kaku haizetsu undō 核廃絶運動 ${ }^{17}$ ) et le mouvement antinucléaire ont en commun leur point de départ, nous devons retrouver les liens qui les rassemblent. Comment les mouvements pour la réduction de l'armée, pour la paix, pour l'abolition de l'arme atomique d'une part et les mouvements antinucléaires d'autre part peuvent-ils se rejoindre ? Telle est la question à laquelle nous devons réfléchir. Jusqu’à présent, les premiers et les seconds militaient séparément. Moi-même, je ne participais pas aux mouvements contre l'arme atomique. Comment relier les deux pour se renforcer?

Historiquement, le problème vient notamment du fait que les deux grandes organisations, le Gensuikin 原水禁18 (Congrès japonais contre les bombes $\mathrm{A}$ et $\mathrm{H}$ ) et le Gensuikyō 原水協 ${ }^{19}$ (Conseil japonais contre les bombes $\mathrm{A}$ et $\mathrm{H}$ ), menaient des actions séparées. Le Gensuikin, dont la base actuelle est le syndicat Rengō 連合 ${ }^{20}$, était un mouvement proche de l'ancien Parti socialiste (Shakaitō 社会党), auquel l'actuel Parti démocrate (Minshutō 民主党) est plus ou moins lié. Mais le Rengō comprend les syndicats ouvriers aussi bien d'entreprises du secteur de l'armement telle que Mitsubishi Heavy Industries Ltd. (Mitsubishi jūkōgyō 三菱重工業) que de fabricants de centrales et d'entreprises du secteur de l'énergie électrique. Les syndicats des plus grandes industries qui sont fédérés au Rengō ont su se maintenir grâce au nucléaire. Le Gensuikin ne pouvait donc que difficilement mener des combats antinucléaires.

Le Gensuikyō, quant à lui, est proche du Parti communiste japonais (Nihon kyōsantō日本共産党). Dans le contexte de la guerre froide, il défendait la légitimité pour les pays de l'Est de posséder l'arme nucléaire, et avait donc fait de l'utilisation pacifique du nucléaire son grand principe. Ces derniers temps, les militants du Gensuikyō ont enfin commencé à utiliser l'expression "retrait du nucléaire " (genpatsu tettai 原発撤退), et ils viendront participer au "Rassemblement pour sortir du nucléaire " (Genpatsu

17. Abréviation de kakuheiki haizetsu undō核兵器廃絶運動 [N.D.T.].

18. Abréviation de Gensuibaku kinshi Nihon kokumin kaigi 原水爆禁止日本国民会議. Il a été créé en 1965 [N.D.T.].

19. Abréviation de Gensuibaku kinshi Nihon kyōgikai 原水爆禁止日本協議会. Il a été créé en 1955 [N.D.T.].

20. Abréviation de Nihon rōdōkumiai sōrengōkai 日本労働組合総連合会. Il s'agit d'un centre national, l'unique au Japon depuis l'union en 1989 de tous les centres divisés, regroupant les fédérations industrielles des syndicats d'entreprises [N.D.T.]. 
ni sayōnara shükai 原発にさようなら集会) du 19 septembre 2011 au parc Meiji pour lequel nous avons lancé l'appel ${ }^{21}$, mais ils sont très en retard. Toutefois, l'idée de l'utilisation pacifique du nucléaire a totalement échoué et il est devenu évident que les seules énergies pacifiques possibles sont les énergies naturelles : cela représente une réelle avancée.

Désormais, la distinction que l'on opérait entre la lutte contre l'arme atomique et la lutte antinucléaire ne peut que disparaittre. Le gouvernement et les milieux économiques envisagent la survie du nucléaire dans les termes d'une "diminution de l'énergie nucléaire ». Personne ne pourra revenir sur le fait que la construction de nouvelles centrales est devenue impossible.

Pour le mouvement antinucléaire, le problème qui va se poser dorénavant est celui de la centrale syndicale Rengō, car c'est elle qui détient le pouvoir dans les mouvements de travailleurs. Comment réduire l'activité des industries qui font du profit avec l'énergie nucléaire et l'armement, telles que les industries de l'énergie électrique, de l'énergie nucléaire, de l'armement, des appareils électriques et l'industrie sidérurgique ? Comment les reconvertir ? Voilà les questions à venir. Concrètement, il faut que le nombre des défenseurs de la sortie du nucléaire augmente dans le secteur industriel. Les cadres du Rengō et les cadres du Parti démocrate ont à peu près le même profil : il faut donc convertir ces deux classes de cadres à l'« industrie de l'énergie pacifique et naturelle ». Cela permettrait de réorienter les actions au sein de la centrale Rengō. Il naîtrait alors un conflit au sein du Parti démocrate opposant les pro-nucléaires et les antinucléaires, avec éventuellement une scission. L'enjeu ici est d'empêcher que le Gensuikin, moteur du mouvement, se retrouve isolé.

D'après moi, dans la mesure où le Gensuikyō, sous influence du Parti communiste, ne dépend ni de la structure du secteur industriel ni des intérêts des entreprises, le conservatisme du Rengō dans le contexte d'une société avançant vers la sortie du nucléaire finira par se révéler au grand jour tandis que le pouvoir du Parti démocrate commencera à s'ébranler, semant

21. Appel lancé par Kamata Satoshi 鎌田慧 et Uchihashi Katsuto 内橋克人 (économiste), Ōe Kenzaburō 大江健三郎 (écrivain), Ochiai Keiko 落合恵子 (écrivain), Sakamoto Ryūichi 坂本龍一 (musicien), Sawachi Hisae 澤地久枝 (journaliste, essayiste), Setouchi Jakuchō 瀬戸内寂聴 (écrivain), Tsujii Takashi 辻井喬 (poète, écrivain), Tsurumi Shunsuke 鶴見俊輔 (philosophe) [N.D.T.]. 
le trouble parmi les sympathisants démocrates. Est-ce qu'un nouveau mouvement porté par des citoyens, dépassant les partis politiques, pourra alors voir le jour ? Si oui, jusqu'où pourra-t-il être influent sur la question de l'arrêt et du démantèlement nucléaire ?

\section{Vers la sortie du nucléaire : trois questions}

Quels sont les problèmes auxquels le mouvement antinucléaire doit dorénavant s'attacher ? Il y a tout d'abord celui des déchets nucléaires.

Jusqu'à la fin des années 1960, les promoteurs du nucléaire soutenaient l'idée d'un avenir radieux grâce à une " énergie propre ", que l'on appelait aussi la " troisième énergie ", ou avançaient l'argument du développement local. C'est le discours qui a servi à la construction des centrales. C'est difficile à croire aujourd'hui, mais à l'époque, on désignait le nucléaire par l'expression " un explosif pour le développement local " (chiiki kaihatsu no kibakuzai 地域開発の起爆剤). Après Three Mile Islands, on a remplacé " explosif " par " avantage » (meritto メリット). On nous promettait que le nucléaire allait permettre à l'économie locale de se développer en engendrant de nouvelles industries auxiliaires. Cela ne s'est produit nulle part. Pour la bonne raison que, les centrales s'installant dans des zones dépeuplées, on n'aurait pas idée d'aménager des terrains pour industrialiser ces zones. C'est bien parce que ces zones ne sont pas aménageables que l'on y construit des centrales. Le nucléaire s'est implanté sur l'illusion, ou la fiction, du développement local.

C'étaient les discours tenus dans les années 1960. Mais avec l'accident de Three Mile Islands, en 1979, est né un doute. La presse changea de ton. Le journal Asahi passa en effet, après l'accident, de "Yes " à "Yes, but ". Plus tard, vers 1983, dans le même journal Asahi, la journaliste Ōkuma Yukiko 大熊由紀子 écrit une longue série d'articles intitulée « Le combustible nucléaire » ("Kakunenryō » 核燃料), que j'ai critiquée en provoquant un débat publié dans le journal Nihon dokusho shinbun 日本読書新聞22.

22. Journal de critique littéraire qui a connu son heure de gloire durant les années 1960 et 1970, offrant ses pages aux grands débats animés par de nombreux intellectuels d'extrême-gauche. Sa publication est suspendue depuis 1984 [N.D.T.]. 
Ensuite, avec l'accident qui s'est produit à Tchernobyl en 1986, on est passé de «Yes, but » à " No, but ", mais sans jamais aller jusqu'au " No ». Seul le journal Tōkyō shinbun 東京新聞 semble aujourd'hui dire : «No».

En fait, c'est depuis Tchernobyl que l'implantation de centrales sur de nouveaux sites est devenue impossible. On a donc augmenté le nombre de réacteurs dans les centrales existantes. Pour ce qui est des centrales de Hamaoka ou de Fukushima, les terrains avaient déjà été achetés, il était donc possible d'y construire de nouveaux réacteurs. À Higashidōri-mura, il y avait également la possibilité physique d'ajouter des réacteurs puisque les terrains avaient été prévus pour accueillir 10 réacteurs de la compagnie Tōhoku denryoku et 10 de Tepco. Mais comment construire 20 réacteurs ! Sans parler des suspicions qui pèsent sur d'éventuels autres usages possibles de ces terrains (comme la construction d'un centre de stockage des déchets ou d'une usine de combustible nucléaire). S'agissant des centrales, non seulement l'implantation sur de nouveaux sites est impossible aujourd'hui mais l'augmentation du nombre de réacteurs sur un site existant l'est devenue également. Il reste à lutter pour l'arrêt des 11 réacteurs encore en marche sur les 54, à empêcher leur remise en activité2 ${ }^{23}$. Nous sommes en surproduction d'énergie électrique.

Il reste à régler le problème des centres de stockage des déchets nucléaires : qu'en faire ? Et les déchets : où les transporter ? Les regrouper à Fukushima? Mais Fukushima est proche de Tokyo. Et que se passe-t-il si on laisse en l'état les barres de combustible usagées de Fukushima Daiichi? Où les transporter?

Jusqu'à présent, lorsque j'allais faire mes enquêtes dans des localités où il y a une centrale, les gens de l'administration ou de la compagnie d'électricité me disaient, avant même que je ne leur pose la question : «Ne vous inquiétez pas, nous transportons les déchets à Rokkasho-mura ». L'existence de Rokkasho-mura rassurait. Or, Rokkasho-mura ne peut pas contenir les déchets de tout le Japon. Il y avait un projet confus de construire un deuxième centre de stockage sur le même site, ou une usine de MOX, mais rien n'est encore clair. Le pays n'est pas en mesure, actuellement, d'élaborer

23. Depuis le 5 mai 2012, il ne reste plus aucun réacteur en marche au Japon. Pour la première fois depuis quarante-deux ans, le pays fonctionne sans énergie nucléaire [N.D.T.]. 
une nouvelle stratégie pour l'avenir administratif du nucléaire. Pour les militants, c'est une chance à saisir, c'est maintenant qu'il faut y mettre toutes nos forces.

Du côté des pro-nucléaires, la logique qui leur reste aujourd'hui est, pour les centrales encore en activité, de les faire fonctionner tant que possible, en renforçant leur sécurité. Et par ailleurs, de maintenir l'industrie en exportant les centrales à l'étranger. Cette tendance ne s'est pas arrêtée. Parmi les pays destinataires de nos centrales, il y a le Vietnam ou la Jordanie ; avec la Mongolie, il existe un projet d'exportation de nos déchets nucléaires contre l'importation de leur uranium. Ce redéploiement vers l'extérieur reproduit ce qui s'était passé au début des années 1980 avec l'exportation de la pollution. À l'époque, le Japon avait déplacé en Asie du Sud-Est ses usines chimiques ou ses cokeries qui ne pouvaient plus être en activité sur son propre territoire. Nous ne pouvons plus accepter aujourd'hui de reproduire ce passé qui a consisté à faire de notre territoire un territoire propre, en exportant la pollution à l'étranger. Le deuxième problème auquel nous devons nous attacher sera ainsi de rompre avec cette exportation de la pollution qu'est l'exportation des centrales et des déchets nucléaires.

Enfin, la question urgente consiste à empêcher la remise en activité des centrales à l'arrêt. La centrale de Hamaoka, en particulier, ne doit en aucun cas être remise en service. Bien que l'arrêt ne règle pas le problème de l'énorme danger que représentent les centrales puisque les barres de combustible demeurent à l'intérieur, il est impératif de les maintenir à l'arrêt. L'objectif est ainsi d'amener les centrales, l'une après l'autre, de l'arrêt pour maintenance à l'arrêt définitif et au démantèlement. Ne pas accepter la remise en service des centrales, les pousser à l'arrêt définitif, tout en empêchant la fuite par l'exportation vers l'étranger : voilà les problèmes auxquels nous devons nous attacher dans l'étape actuelle pour sortir du nucléaire.

\section{La visibilité de la structure discriminatoire}

Depuis l'accident nucléaire de Fukushima, les travailleurs exposés aux rayonnements sont devenus visibles. Cela a un impact considérable sur la population. Jusqu'alors, on ne voyait jamais ces personnes contaminées 
par le travail dans des centrales. Aujourd'hui encore, on ne les voit pas complètement, mais la population a pris conscience de l'existence de ces travailleurs qui doivent rester dans la centrale pour réparer les dégâts après l'accident. On sait aujourd'hui qu'il y a de nombreux cas de contamination, y compris chez les femmes, et on s'inquiète de l'irradiation qui a pu toucher les travailleurs sur place au moment des explosions, les pompiers et les soldats des Forces d'auto-défense venus éteindre les feux et porter secours. Certains l'ont certainement été.

On commence aussi à savoir, dans la structure d'ensemble de l'organisation du travail de l'industrie nucléaire, les positions atroces auxquelles sont relégués les travailleurs des centrales. On sait par exemple qu'on y vient travailler pour 50000 yens $^{24}$, ou qu'il y a eu des cas d'hyperthermie par excès de travail. On sait aussi - bien que Tepco le nie - qu'il y a eu, en plus des cas d'accident mortel, des cas de contamination aiguë. La notion de « travail exposé aux rayonnements " (hibaku rōdō 被曝労働) est ainsi apparue de manière très concrète, c'est un fait considérable car il s'agit d'un problème humain très important.

Bien sûr, il y a eu des travailleurs contaminés avant l'accident de Fukushima. Tous ceux qui se sont approchés du cœur d'un réacteur lors d'un contrôle périodique de maintenance ont été contaminés. Ils ont développé par la suite des leucémies ou des cancers. Pourtant, nous n'avons pas pu mener de combat pour que la maladie professionnelle soit reconnue. Pour mener une action en justice, il faut des avocats, des fonds, et des adresses fixes, ce que n'ont pas les travailleurs du nucléaire dont la vie est instable. Nous n'avons pas pu aller au tribunal. À ma connaissance, il n’y a que treize travailleurs dont les troubles ou les décès liés au nucléaire ont été reconnus comme accident du travail. Parmi eux, deux sont des victimes de l'accident de la compagnie JCO qui en sont décédées. Très peu de cas sont reconnus, le reste est couvert d'un voile opaque. Avec l'accident de Fukushima, on a cependant rendu public ces problèmes. Qu'une partie de

24. Sur ces 50000 ou 60000 yens de salaire journalier facturé, le travailleur ne touche en réalité que de 7000 à 11000 yens car les différents niveaux de sous-traitants en ponctionnent une bonne partie. Voir Nadine et Thierry Ribault, Les Sanctuaires de l'abîme. Chroniques du désastre de Fukushima, Paris, Éditions de l'Encyclopédie des Nuisances, 2012, p. 20 [N.D.T.]. 
l'opinion publique ait souhaité, afin de préserver les plus jeunes, que seuls les hommes âgés de plus de soixante ans soient envoyés à Fukushima faire le "sale boulot " signifie qu'elle a pris conscience des dangers du travail dans une centrale nucléaire.

Cependant, comme la situation de l'emploi est critique, un travail de deux à trois heures par jour rémunéré 20000 ou 30000 yens attire encore du monde. Combien de temps cela va-t-il durer ? Si nous portons sur le devant de la scène les problèmes relatifs au travail exposé aux rayonnements, le nombre de travailleurs qui acceptent d'occuper le dernier échelon de la sous-traitance du travail baissera. Cela constituera, à n'en pas douter, un obstacle à la poursuite du nucléaire. De plus, il est peu probable que le nombre de nouveaux employés dans les compagnies d'électricité augmente, et dans les universités, de moins en moins d'étudiants choisiront la section des sciences nucléaires, ce qui était déjà une tendance constatée ces derniers temps. Le nucléaire connaît un tournant avec cet accident qui le précipite dans le crépuscule de son histoire.

M. Masukura, de la ville voisine de la centrale de Fukushima, a commencé à lutter contre les centrales nucléaires parce qu’il y avait travaillé. Les pêcheurs de l'île d'Innoshima 因島 qui s'opposent à la centrale de Kaminoseki 上関 m'ont raconté aussi qu'ils étaient devenus antinucléaires après y avoir travaillé en tant que journaliers. Ces expériences vécues vont commencer à se transmettre de diverses façons. Les médias commencent aussi, enfin, à s'intéresser aux travailleurs contaminés. On parle des problèmes liés au travail dans les centrales, des travailleurs qui viennent sous un faux nom ${ }^{25}$, des mineurs, des employés sans dosimètre.

En fait, c'est toute l'industrie nucléaire qui s'était construite sur une structure discriminatoire. Celle-ci apparaissait aussi dans l'organisation du travail qui est dépendante des employés situés en bas de l'échelle. Les employés permanents des compagnies d'électricité et des grandes entreprises générales de construction travaillent dans des lieux sûrs - même s'il y a sans doute eu des cas de contamination parmi eux - tandis que la plupart des travailleurs exposés aux rayonnements étaient recrutés par des sous-

25. Le contrat des travailleurs du nucléaire est renouvelé jusqu'à ce qu'ils aient reçu la dose de radioactivité maximale annuelle autorisée ; certains viennent ainsi se faire réembaucher sous un faux nom. Voir Nadine et Thierry Ribault, op. cit., p. 20 [N.D.T.]. 
traitants de sixième ou de septième degré. On peut dire également que ce sont les sites placés au bas de l'échelle du territoire national qui ont été choisis pour installer les centrales. Il suffit de regarder la carte des centrales de Tepco : pas un seul réacteur dans la région du Kantō. Par contre, il y en a 7 dans le département de Niigata, 10 dans le département de Fukushima et 1 en construction dans le département d'Aomori, cela fait 18 réacteurs au total. En ajoutant à ce compte les 6 réacteurs de la centrale de Tomari 泊 de la compagnie d'électricité du Hokkaidō et de la centrale d'Onagawa 女 川 de la compagnie du Tōhoku, cela fait au total 24 réacteurs, soit presque la moitié des 54 réacteurs que totalise le pays. Et si on ajoute les réacteurs de la baie de Tsuruga, ils représentent plus de la moitié du total. Les centrales ont été construites dans les régions pauvres face à la mer du Japon, comme celle où est né Minakami Tsutomu 水上勉 ${ }^{26}$, et dans les régions pauvres du Tōhoku.

"On a tout eu chez nous, sauf les mines d'uranium ", m'avait très justement confié, avec un brin de cynisme, un employé de la Ville de Mutsu. En effet, à part les mines d'uranium, le département d'Aomori a accueilli tous les équipements du nucléaire. Comme je l'ai écrit dans Reportage sur la presquîlle nucléaire de Shimokita, le milieu financier et l'État ont mené de concert ce projet - si l'on peut dire - de nucléarisation de la presqu'île. La politique nucléaire est ainsi apparue en ciblant les localités les plus vulnérables. Ce sont des territoires où il est facile de s'imposer, d'attaquer, de faire accepter, de rendre dépendants. Les centrales japonaises sont installées aux points d'intersection entre la structure discriminatoire du travail et celle du territoire.

\section{Quelles leçons du passé ?}

Revenons à la " pensée du refus».

"Si nous avons la mer et les champs, cela nous suffit pour vivre. " Ces mots que m’a confiés la militante Kumagai Asako contre la centrale d'Ōma

26. Écrivain japonais (1919-2004) d'origine modeste, né à Ōi 大飯 où a été construite une centrale nucléaire en 1979. Il est célèbre pour ses romans à suspense sur des thèmes sociaux, comme la maladie de Minamata [N.D.T.]. 
sont précieux. Au-delà de la problématique du mouvement antinucléaire, ils relèvent d'une philosophie de l'humanité. L'homme ne peut vivre s'il n'a plus de terres pour cultiver et si la mer est polluée. À l'inverse, l'homme possède l'expérience d'une vie exclusivement basée sur les produits de la terre et de la mer. C'est indéniable. En nous les confisquant, l'État et les compagnies d'électricité nous ont tenu un mensonge, nous promettant une vie prospère grâce aux centrales et à l'industrialisation. Or, l'accident de Fukushima nous a révélé que cette vie était impossible. L'heure n'est-elle pas venue de nous confronter à cette pensée ?

M. Masukura disait aussi : "Les paysans ne se laissent pas berner". L'État et les industries ont réuni tous leurs efforts pour leur asséner que leur mode de vie était du passé et qu'il ne correspondait plus à la vie urbaine, pour transformer ces populations en force de travail au service de la ville. Nous pouvions pourtant vivre autrement. Cela peut être une leçon, non seulement pour le Japon, mais aussi pour les autres pays.

Quand je suis allé à Fukushima au mois de mai, je me suis rendu dans le village d'Iitatemura 飯舘村. C'était la saison du repiquage, mais il n'y avait personne dans les rizières. On ne voyait que les restes de la moisson précédente, les pieds de riz, tout gris, qui avaient pourri. On ne voyait rien du paysage habituel en cette saison, les rizières labourées, puis irriguées, les plants de riz si bien alignés, caressés par le vent. Que sont devenus les grenouilles, les vers de terre et les cigales, tous ces habitants sous terre ${ }^{27}$ ? De nombreux animaux, des bœufs, des porcs, sont également morts. La nature dans cette zone a été complètement détruite par l'accident nucléaire.

Il n'y a que les hommes politiques et les patrons qui peuvent, tout en ayant cela sous leurs yeux, continuer à dire : "Il faut du nucléaire ". Ils sont esclaves de l'argent. Ils sont désormais minoritaires. Ils ne le savent pas encore. Ils croient encore pouvoir donner la priorité à l'économie, convaincre la population avec un tel discours. Ils se trompent. Qu'allons-nous faire de la vie des hommes et de tous les êtres vivants, y compris les microorganismes ? Telle est la question essentielle qui se pose à nous aujourd'hui.

27. Les grenouilles passent leur hiver sous terre, où les larves des cigales se développent aussi [N.D.T.]. 
Il est absolument impossible de continuer le nucléaire. Comment en sortir avant qu'un autre accident ne se produise ? À n'en pas douter, c'est une question qui va devenir centrale dans la politique à venir.

Traduit par Jennifer HASAE

\section{Bibliographie citée de Kamata Satoshi}

1970

Kakusareta kōgai 隠された公害

(Cette pollution cachée),

éd. San.ichi shinsho 三一新書.

\section{1}

Shi ni taeta fūkei 死に絶えた風景

(Un paysage disparu à jamais),

éd. Daiyamondosha ダイヤモンド社.

1991

Rokkasho-mura no kiroku

六ヶ所村の記録 (Chroniques

de Rokkasho-mura), 2 vol.,

Iwanami shoten 岩波書店.

2001

Genpatsu rettōo iku 原発列島を行く

(Parcourir l'archipel nucléaire),

éd. Shūeisha 集英社.

\section{1}

Rupo: Shimokita kaku hantō

ルポ——北核半島 (Reportage

sur la presqu'île nucléaire de Shimokita), écrit avec Saitō Mitsumasa 斉藤光政， Iwanami shoten 岩波書店.

\section{1 [1982]}

Nihon no genpatsu kiken chitai

日本の原発危険地帯 (Les zones nucléaires dangereuses du Japon), [1 ${ }^{\text {re édition }}$ 1982, éd. Ushio shuppansha 潮出版社], réédition augmentée, éd. Seishisha 青志社.

\section{1}

«Genpatsu kyozetsu no shisō to undōo, ima koso» 原発拒絶の思想と運動を、今こそ (Penser le refus du nucléaire et nous mobiliser, ici et maintenant), Sekai 世界, septembre, $n^{\circ} 821$. 